\title{
Plasma-Wall Interaction Studies in the Full-W ASDEX Upgrade during Helium Plasma Discharges
}

A Hakola ${ }^{1} \ddagger$, S Brezinsek ${ }^{2}$, D Douai $^{3}$, M Balden ${ }^{4}$, V Bobkov ${ }^{4}$, D Carralero $^{4}$, H Greuner ${ }^{4}$, S Elgeti ${ }^{4}$, A Kallenbach ${ }^{4}$, K Krieger ${ }^{4}$, G Meisl ${ }^{4}$, M Oberkofler ${ }^{4}$, V Rohde ${ }^{4}$, P Schneider $^{4}$, T Schwarz-Selinger ${ }^{4}$, A Lahtinen ${ }^{5}$, G De Temmerman ${ }^{6}, \mathbf{R}$ Caniello $^{7}$, F Ghezzi ${ }^{7}$, T Wauters ${ }^{8}$, A Garcia-Carrasco ${ }^{9}, \mathbf{P}$ Petersson $^{9}$, I Bogdanovic Radovic ${ }^{10}$, Z Siketic ${ }^{10}$, ASDEX Upgrade Team, EUROfusion MST1 $\S$

${ }^{1}$ VTT Technical Research Centre of Finland Ltd., P.O. Box 1000, 02044 VTT, Finland

${ }^{2}$ Forschungszentrum Jülich GmbH, Institut für Energie- und Klimaforschung Plasmaphysik, Partner of the Trilateral Euregio Cluster (TEC), 52425 Jülich, Germany

${ }^{3}$ CEA, IRFM, 13108 St. Paul Lez Durance Cedex, France

${ }^{4}$ Max-Planck-Institut für Plasmaphysik, Boltzmannstrasse 2, 85748 Garching, Germany

${ }^{5}$ University of Helsinki, Department of Physics, Association EURATOM-Tekes, P.O. Box 43, 00014 University of Helsinki, Finland

${ }^{6}$ ITER Organization, 13067 St. Paul Lez Durance Cedex, France

${ }^{7}$ Istituto di Fisica del Plasma - CNR, Via. Cozzi 53, 20125 Milan, Italy

${ }^{8}$ Laboratory for Plasma Physics, ERM/KMS, Partner of the Trilateral Euregio Cluster (TEC), 1000 Brussels, Belgium

${ }^{9}$ Department of Fusion Plasma Physics, Royal Institute of Technology, 10044 Stockholm, Sweden

${ }^{10}$ Rudjer Boskovic Institute, P.O. Box 180, 10002 Zagreb, Croatia

E-mail: antti.hakola@vtt.fi

\begin{abstract}
Plasma-wall interactions have been studied in the full-W ASDEX Upgrade during its dedicated helium campaign. Relatively clean plasmas with a $\mathrm{He}$ content of $>80 \%$ could be obtained by applying Ion Cyclotron Wall Conditioning (ICWC) discharges upon changeover from D to He. However, co-deposited layers with significant amounts of $\mathrm{He}$ and $\mathrm{D}$ were measured on $\mathrm{W}$ samples exposed to ICWC plasmas at the low-field side (outer) midplane. This is a sign of local migration and accumulation of materials and residual fuel in regions shadowed from direct plasma exposure albeit globally D was removed from the vessel. When exposing $\mathrm{W}$ samples

$\S$ See the author list of "Overview of progress in European Medium Sized Tokamaks towards an integrated plasma-edge/wall solution" by H Meyer et al, to be published in Nuclear Fusion Special issue: Overview and Summary Reports from the $26^{\text {th }}$ Fusion Energy Conference (Kyoto, Japan, 17-22
\end{abstract}

$\ddagger$ Corresponding author October 2016) 
to ELMy H-mode helium plasmas in the outer strike-point region, no net erosion was observed but the surfaces had been covered with co-deposited layers mainly consisting of $\mathrm{W}, \mathrm{B}, \mathrm{C}$, and $\mathrm{D}$ and being the thickest on rough and modified surfaces. This is different from the typical erosion-deposition patterns in D plasmas, where usually sharp net-erosion peaks surrounded by prominent net-deposition maxima for $\mathrm{W}$ are observed close to the strike point. Moreover, no clear signs of W nanostructure growth or destruction could be seen. The growth of deposited layers may impact the operation of future fusion reactors and is attributed to strong sources in the main chamber that under suitable conditions may switch the balance from net erosion into net deposition, even close to the strike points. In addition, the absence of noticeable chemical erosion in helium plasmas may have affected the thickness of the deposited layers. Retention of He, for its part, remained small and uniform throughout the strike-point region although our results indicate that samples with smooth surfaces can contain an order of magnitude less He than their rough counterparts.

Keywords: plasma-wall interaction, helium, tungsten fuzz, erosion, retention

\section{Introduction}

ITER has selected tungsten (W) to be used in the plasma-facing components (PFCs) of its divertor structures due to the good power-handling capabilities of $\mathrm{W}$, low physical sputtering of the wall structures by plasma bombardment, and small retention of radioactive tritium (T) in the PFCs [1]. So far, the majority of plasma-wall interaction studies in tokamaks with partial or full W coverage, including JET [2] and ASDEX Upgrade (AUG) [3], have been carried out in hydrogen $(\mathrm{H})$ or deuterium (D) plasmas but recently also helium (He) has gained increased research interest. This is due to the possible start-up phase of ITER with helium, as well as to alpha particles produced in D-T fusion reactions in the active phase of ITER, setting the need to understand in detail the interaction processes between $\mathrm{W}$ and He.

The most important research topic is understanding the erosion, re-deposition, surface modification, and retention characteristics of W PFCs in different types of He plasmas. In addition, a smooth start of plasma operations in helium requires cleaning the vessel wall from residual fuel species (e.g., $\mathrm{H}$ and D) of earlier discharges as well as from various impurities (such as nitrogen $(\mathrm{N})$ and oxygen $(\mathrm{O})$ or, in the case of AUG, also boron (B) and carbon (C)) [4]. Compared to operations in $\mathrm{H}$ or D, during He plasma exposure nanoscale structures may develop on W-based wall components if the surface temperature, $T_{\mathrm{S}}$, is high enough (but stays well below $2000 \mathrm{~K}$ to prevent $\mathrm{W}$ from recrystallizing and thus the PFCs from losing their hardness and strength [1]), the impact energy of $\mathrm{He}$ ions is $E_{\text {in }}>20 \mathrm{eV}$, and sufficiently large fluence, $\phi$, is accumulating on the PFCs [5,6]. Under such conditions, helium starts inducing bubbles in W, which will modify the surface in the nanometer scale, finally resulting in the formation of a porous surface layer with coral-like tendrils, referred to as fuzz, as the surface temperature exceeds $1000 \mathrm{~K}$ and the fluence increases above $1-2 \times 10^{24} \mathrm{He}^{+} \mathrm{m}^{-2}$. 
Critical questions are whether these surface modifications take place during ELMy H-mode operations of ITER, have a negative impact on the plasma performance or the power-handling capabilities of the PFCs, or result in the generation excess amounts of dust in the vessel; these concerns stem from recent observations that the nanostructured layers with He bubbles can be extremely fragile and may strongly reduce the surface thermal conductivity of PFCs. Equally important is to clarify how the retention characteristics of the wall structures are altered. Interestingly, laboratory experiments indicate retention to be reduced both when exposing $\mathrm{W}$ samples to He-seeded $\mathrm{H}$ or $\mathrm{D}$ plasmas or when pre-treating the $\mathrm{W}$ surfaces with He before their exposure to H/D plasmas [7-9]. This is attributed to the above-mentioned formation of nanoscale He bubbles which act as a diffusion barrier close to the surface, preventing hydrogen from penetrating deep into the bulk. However, the situation may be different in a reactor environment where thick D- or T-rich co-deposited layers can form, being the most prominent on rough and modified surfaces [10].

The generation of fuzz has been experimentally observed in several linear plasma devices $[5,6,11,12]$ while identifying the onset of fuzz formation in tokamaks and temporal evolution of the modified surface layers during the plasma exposure are now a subject of joint experiments in different facilities. Pioneering work has been carried out in the TEXTOR [13], Alcator C-Mod [14], and DIII-D [15] tokamaks. The TEXTOR experiments have revealed that the flux of low- $\mathrm{Z}$ impurities is an additional factor in determining whether the $\mathrm{W}$ nanostructures are grown, eroded by the impurity ions, or even covered with co-deposited layers - even if all the criteria listed above for the formation of nanoscale surface modifications were fulfilled. The TEXTOR results agree with the data from the PISCES-B linear facility where W samples have been exposed to Be-seeded He plasmas, and already a $0.2-\%$ impurity concentration in the plasma has been noticed to reduce the growth of the nanostructured layer on the surface [16]. Additional support comes from Alcator C-Mod where the almost complete absence of low-Z impurities have made it possible to stimulate nanostructure growth during Lmode plasma discharges [14]. The DIII-D experiments, for their part, have shown that transient power loads up to $0.1 \mathrm{MJ} \mathrm{m}^{-2}$ in He plasmas do not lead to damage or roughening of the exposed $\mathrm{W}$ surfaces. This was the case for both virgin samples and for samples pre-loaded in the linear plasma facility PISCES-A with a pure He beam [15]. On the other hand, reduced gross erosion was measured for samples having pre-formed fuzz on them while after the experiment an almost 30-nm deep layer consisting of nanobubbles was identified on all the samples, largely independent of the applied fluence. These observations suggest that during H-mode operations the He-induced nanostructured layer may not necessarily affect the lifetime of the PFCs.

In this contribution, we discuss the lessons learnt from two plasma-wall interaction experiments, carried out in AUG during its dedicated helium campaign in 2015. The main focus points are the efficiency of Ion Cyclotron Wall Conditioning (ICWC) in reducing the amount of deuterium in the AUG vessel upon changeover from D to He plasma operations as well as determining the erosion, deposition, fuel retention, and 
surface modification patterns of different W samples, some of them being pre-modified by a helium beam in the high heat-flux device GLADIS, resulting from exposure to a series of $\mathrm{H}$-mode discharges in He. It is worth mentioning that, after this experiment, advances have been made in developing ITER-relevant scenarios and efficiently controlling edgelocalized modes (ELMs) using resonant magnetic perturbations under a large range of pedestal collisionalities. Interestingly, fuelling efficiency appears to be much higher in He than in D plasmas in AUG, which, accompanied with different wall recycling characteristics of $\mathrm{D}$ and $\mathrm{He}$, results in lower divertor pressures at comparable pedestal pressures and hotter divertor plasmas, thus influencing PWI processes.

The article is organized as follows. Section 2 discusses the ICWC method and presents the results of the cleaning experiment carried out. In section 3, erosion, deposition, and surface modifications of the $\mathrm{W}$ samples after the H-mode plasma experiment are reported while section 4 concentrates on the retention of He on the analyzed W samples. Finally, conclusions are drawn and further steps are outlined in section 5 .

\section{Start-up of helium plasma operations in AUG}

Depending on the plasma operations carried out in a fusion device, the first wall may need to be regularly conditioned to assist in obtaining breakdown during subsequent plasma discharges or in recovering from disruptions. Wall conditioning can also remove $\mathrm{T}$ from the reactor and clean the vessel walls from residual fuel inventories when switching from one plasma gas to another. Typically, conditioning is done by DCglow discharges but this approach cannot be used in future reactors where the magnetic field will be kept continuously switched on. To this end, ICWC is a good candidate, and it has already been tested in, e.g., JET, AUG, TEXTOR, and Tore Supra [4,17]. In ICWC, low-temperature (electron temperature $T_{\mathrm{e}}<10 \mathrm{eV}$ ) and low-density (electron density $n_{\mathrm{e}}=10^{16}-10^{18} \mathrm{~m}^{-3}$ ) plasmas are formed resulting in a substantial flux of charge-exchange (CX) neutrals onto the wall structures. Such particles can mobilize the fuel and impurity atoms residing in the material, resulting in removal rates up to $10^{17} \mathrm{D} \mathrm{m}^{-2} \mathrm{~s}^{-1}$ [17]. For comparison, typical densities and temperatures in glow discharge plasmas are $10^{15} \mathrm{~m}^{-3}$ and $1-10 \mathrm{eV}$.

At JET, $\mathrm{D}_{2}$-ICWC discharges have been applied after loading the walls with hydrogen, and they are able to increase the $\mathrm{D}$ content of the plasma from $\mathrm{D} /(\mathrm{D}+\mathrm{H})=0.3$ to 0.5 . However, less than $10 \%$ of the $\mathrm{H}$ inventory could be removed from the walls by this isotope-exchange technique while retention of D from the ICWC plasma was almost three times higher. The fuel can also be removed by He-ICWC, and again the results from JET indicate D being efficiently removed but almost $80 \%$ of the injected He being retained in the vessel. At Tore Supra, for its part, pulsed ICWC discharges $\left(\mathrm{H}_{2}\right.$, pulse duration $<1 \mathrm{~s}$ ) have been carried out and retention has been reduced well below the contribution of pumping, without compromising the cleaning rate on the walls.

So far, however, data on the applicability of ICWC in cleaning the vessel walls from 
fuel-containing layers during changeover from $\mathrm{H}$ or $\mathrm{D}$ to He plasmas is rather scarce. This deficiency has now been addressed at AUG during a dedicated He-ICWC experiment before the onset of the 2015 helium campaign. In the experiment [18], two ion cyclotron resonance heating $(\mathrm{ICRH})$ antennas were used during 20 discharges at $2.0 \mathrm{~T}$, having a total duration of $180 \mathrm{~s}$. The RF power was $100-200 \mathrm{~kW}$ at $30 \mathrm{MHz}$ and He was injected into the torus from the outer midplane such that the pressure was $1-5 \times 10^{-4}$ mbar. The fuel content of the vessel was monitored by (i) determining the evolution of the $\mathrm{D}$ and He content of standard AUG plasma discharges in He before, during, and after ICWC by measuring the CX flux of neutrals by neutral particle analysers (NPA), (ii) evaluating the temporal evolution of the $\mathrm{D}_{\alpha}(656.1 \mathrm{~nm})$ and He I $(667.8 \mathrm{~nm})$ spectral lines in the divertor and main-chamber regions, (iii) studying the composition of the exhaust gas using mass spectrometry, and (iv) measuring the D and He contents of W samples exposed to plasmas in the outer midplane.

Significant release of D from the wall structures could be observed during ICWC. This we notice from the CX data for neutral D flux (see figure 1a), which show a peak in the beginning of the ICWC discharges as the heating power in increased to $200 \mathrm{~kW}$ and a gradual decrease towards the end of the experiment. Similar trend was observed by spectroscopic measurements. The release rate was quantified on the basis of mass spectrometry. From the temporal behaviour of the partial pressures of $\mathrm{H}_{2}, \mathrm{HD}$, and $\mathrm{He}+\mathrm{D}_{2}$ signals during each discharge (figure $1 \mathrm{~b}$ ) the total amount of $\mathrm{D}$ being removed by ICWC integrates to $\sim 13 \mathrm{~Pa} \mathrm{~m}^{3}$, including 15 min outgassing between discharges (phase $\mathrm{C}$ in figure 1c). This corresponds roughly $3.1 \times 10^{21} \mathrm{D}_{2}$ molecules at $300 \mathrm{~K}$ and, thus, an average removal rate of $1.7 \times 10^{19} \mathrm{D}_{2}$ molecules $\mathrm{s}^{-1}$. During overnight and weekend outgassings (phases $\mathrm{B}, \mathrm{D}$, and $\mathrm{F}$ ) the amount remained $<1 \mathrm{~Pa} \mathrm{~m}^{3}$ while during two H-mode plasma discharges in helium before ICWC (phase A) and during $25 \mathrm{H}$-mode discharges after ICWC (phase E, see section 3) $\mathrm{D}_{2}$ release of $\sim 3 \mathrm{~Pa} \mathrm{~m}^{3}$ was measured. By taking into account the plasma time for these two phases $(8 \mathrm{~s}$ for phase A, $160 \mathrm{~s}$ for phase $\mathrm{E}$ ), the removal rates would be $8.9 \times 10^{19}$ and $0.44 \times 10^{19} \mathrm{D}_{2}$ molecules s ${ }^{-1}$, indicating that the cleaner the vessel, the more time it would take to remove the residual deuterium by repetitive plasma discharges. The He content of the plasma, on the other hand, increased from $\mathrm{He} /(\mathrm{He}+\mathrm{D}+\mathrm{H})=0.4$ (phase A) to 0.8 (phase E). Close to the end of phase $\mathrm{E}$, the $\mathrm{D}$ content of the plasma saturated at a constant level of $\sim 10 \%$ and slowly decreased to $<5 \%$ by the end of the 2-week-long He campaign on AUG.

The efficiency of ICWC was also estimated by surface analyses of bulk W samples before and after their exposure to the ICWC discharges in the low-field side midplane of AUG (figure 1d) [19]. The samples were studied using Elastic Recoil Detection Analysis (ERDA) with a $10-\mathrm{MeV}^{28} \mathrm{Si}^{3+}$ beam for the detection of He and D and Time-of-Flight ERDA with a $36-\mathrm{MeV}^{127} \mathrm{I}^{8+}$ beam to determine the concentrations of heavier impurities. As a result of ICWC, co-deposited layers containing D, B, C, N, and O were formed on all the samples, with concentrations in the range of $0.2-1.5 \times 10^{20}$ atoms $\mathrm{m}^{-2}$. Boron originates from regular boronizations of the AUG vessel, nitrogen from seeding experiments carried out in AUG, while $\mathrm{C}$ is most likely released from PFCs with 

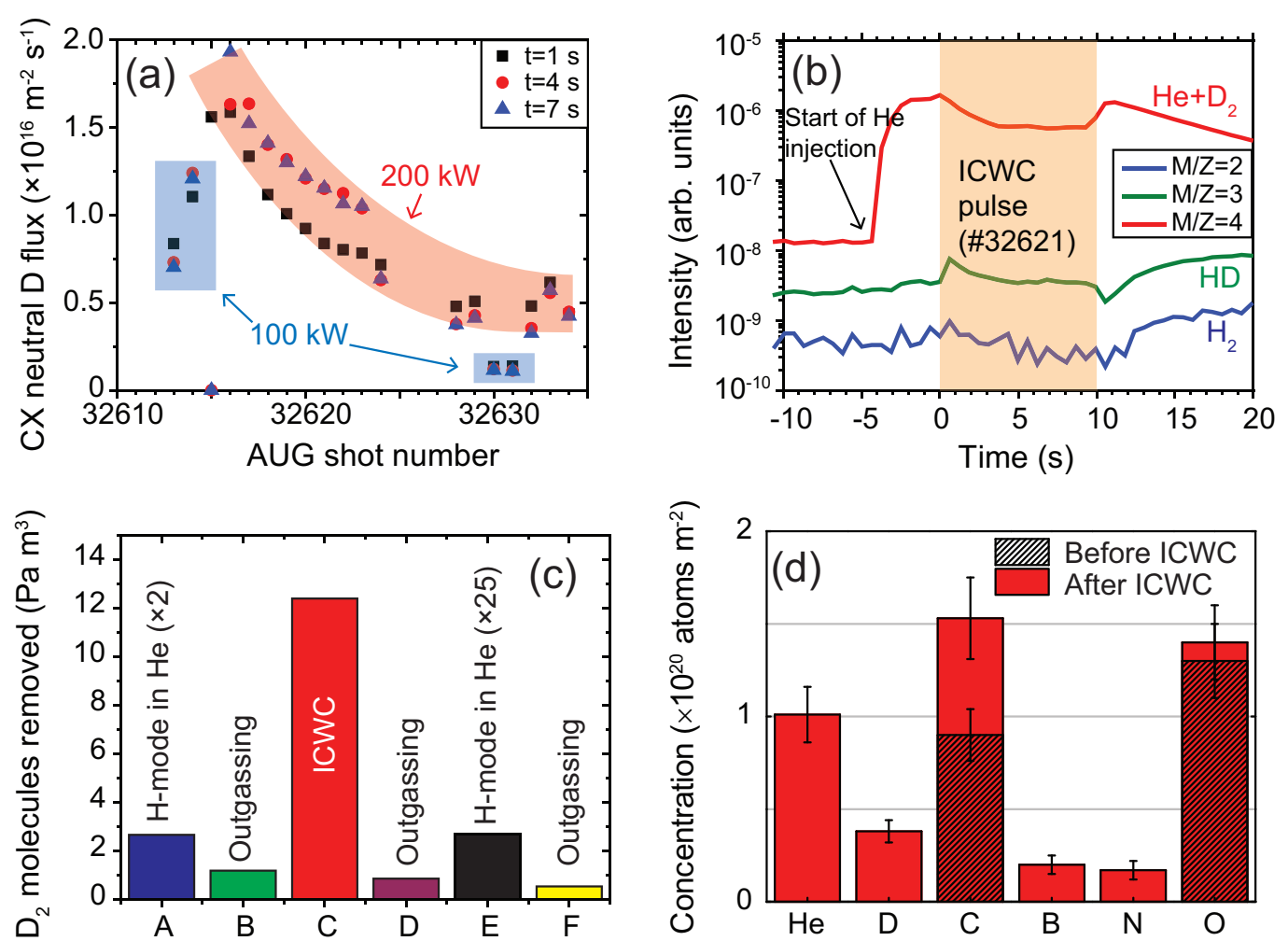

Figure 1. (a) Evolution of the CX neutral D flux during the ICWC experiment at different time points $(1 \mathrm{~s}, 4 \mathrm{~s}$, and $7 \mathrm{~s})$ from the beginning of the discharge; each shot had a duration of $\sim 9 \mathrm{~s}$. The flux has been integrated over the energy and the solid angle, and the data points corresponding to $100 \mathrm{~kW}$ and $200 \mathrm{~kW}$ of $\mathrm{RF}$ power have been grouped under the blue and red regions, respectively. (b) Time traces for selected $M / Z$ signals before, during, and after an ICWC pulse for the AUG shot \#32621. (c) Number of $\mathrm{D}_{2}$ molecules removed during $\mathrm{H}$-mode shots in helium (A and E), overnight and weekend outgassings (B, D, and F), and the actual ICWC experiment $(\mathrm{C})$. The overall duration of the measurement period was 170 hours. (d) Surface densities for selected elements on $\mathrm{W}$ samples at the outer midplane before and after the ICWC experiment

damaged $\mathrm{W}$ coatings on graphite substrates. It is worth noting that the initial $\mathrm{C}$ content of the W surfaces was relatively high, $10^{20} \mathrm{C} \mathrm{m}^{-2}$, thus the net amount of $\mathrm{C}$ deposited on the surfaces is similar or slightly larger than the surface density of $\mathrm{D}$; the origin for the high initial carbon surface contamination is under investigation at the moment. The helium content of the co-deposits, for its part, is a factor of two larger than the amount of $\mathrm{D}$ measured in them, up to $10^{20} \mathrm{He} \mathrm{m}^{-2}$ (see figure $1 \mathrm{~d}$ ). This suggests that, during ICWC, He may substitute a substantial fraction of hydrogen atoms retained on the samples.

One should, however, keep in mind that the analysed samples reflect a situation only at a particular location of the vessel, here at the outer midplane. As discussed above, the data in figure 1 show that globally D is removed from PFCs but, besides being pumped away from the torus, the removed particles can migrate in the ICWC plasma 

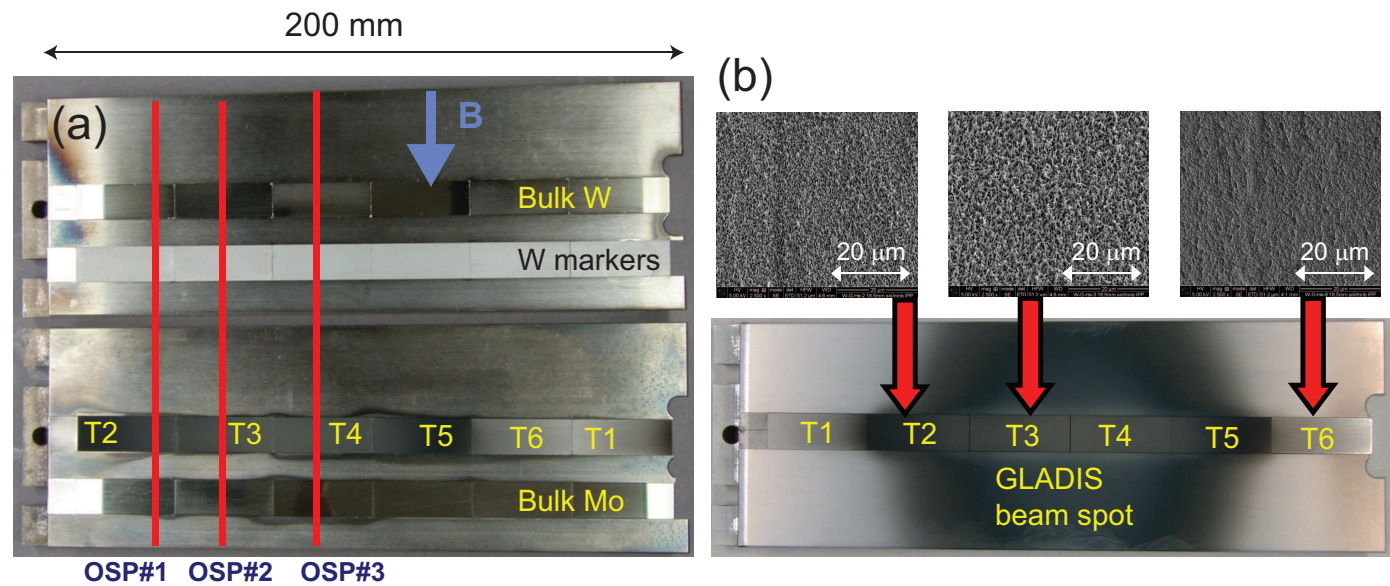

Figure 2. (a) Photograph of the 4 sample types mounted in the divertor target tiles after the plasma experiment. The pre-modified samples are labelled with symbols T1-T6 and the OSP positions for the three phases of the plasma experiment have been marked with red lines. The private flux region is located on the very left. (b) Photograph of the pre-modified samples T1-T6 after their exposure in GLADIS together with SEM images of the modified surfaces of T2, T3, and T6.

and accumulate in shadowed areas of the torus through multiple erosion-deposition steps [20].

\section{Erosion, deposition, and surface modifications of W PFCs at AUG}

\subsection{Samples and their characterization}

The interaction of tungsten PFCs with helium was investigated by exposing four poloidal rows of different samples to ELMy H-mode discharges in He at the outer strike-point (OSP) region of the AUG divertor [22]. The samples were mounted on two bulk W target tiles - two rows per target tile and 6 samples in each row - which were transferred to the desired location using the upgraded divertor manipulator (DIM-II) of AUG [23], see figure 2a.

For each row, a different sample type was selected. On the first tile, bulk W samples were mounted at the centre, while W-coated (thickness $30 \mathrm{~nm}$ ) graphite marker samples were positioned magnetically downstream of them. On the second target tile, the centre row consisted of W samples pre-modified by He exposure in GLADIS [24] (denoted by T1-T6) while in the edge row bulk Mo samples had been positioned. The marker samples were used to determine erosion of $\mathrm{W}$ during the plasma experiment, the purpose of the Mo samples was to estimate re-deposition of $\mathrm{W}$, while the two sets of $\mathrm{W}$ samples provided information on the changes in the surface morphology as well as the formation or erosion of fuzz and retention of He in them.

The pre-modified samples contained a variety of nanostructures on the surfaces with a layer thickness of some $2-3 \mu \mathrm{m}$. The nanostructures were produced by mounting bulk 
W samples in GLADIS on a target plate and exposing them to a pure He beam with an energy of $37 \mathrm{keV}$. A photograph of the sample surface after irradiation is shown in figure 2b. At the centre of the holder, the surface temperature was $\sim 2300 \mathrm{~K}$ and the He fluence around $1.0 \times 10^{24} \mathrm{He}^{+} \mathrm{m}^{-2}$, while at the edge the corresponding values were $1300 \mathrm{~K}$ and $0.4 \times 10^{24} \mathrm{He}^{+} \mathrm{m}^{-2}$. The fluence and surface temperature clearly favoured nanostructure formation while the energy was much higher (in the keV range) compared to the typical values in tokamak edge plasmas (a few eV). Nevertheless, based on microscopy analyses, also at these high energies tendril-like networks had been formed on practically all the samples [24]. At the center (samples T2-T5), the nanoscale network largely resembled fully developed fuzz while in the peripheral zone the surface modifications were not that distinct. The thickness of the modified layer on samples T3 and T4 was approximately $2 \mu \mathrm{m}$ and presumably contained significant helium inventories from the GLADIS treatment (not quantified, however).

The most extensively modified samples T3 and T4 were mounted closest to the OSP positions during the experiment while the samples with more benign surface modifications towards the upper edge of the target tile. The surface morphology of all the samples was monitored by scanning electron microscopy (SEM) and focused ion beam (FIB) measurements. The erosion of the marker coatings was determined by measuring their thicknesses using Rutherford Backscattering Spectroscopy (RBS) with 2.0- $\mathrm{MeV}{ }^{4} \mathrm{He}^{+}$ions before and after the plasma experiment. All the samples were also measured by Nuclear Reaction Analysis (NRA), where $3.8-\mathrm{MeV}^{3} \mathrm{He}^{+}$ions were applied to determine the deposition of different elements, primarily $\mathrm{D}, \mathrm{B}, \mathrm{C}$, and $\mathrm{N}$ on the surfaces. The helium content of the samples is discussed in section 4 .

\subsection{Plasma conditions during sample exposure}

The W samples were exposed to lower single-null (LSN) plasma discharges on AUG with the following parameters: plasma current $I_{\mathrm{p}}=0.8 \mathrm{MA}$, toroidal magnetic field $B_{\mathrm{t}}=2.5 \mathrm{~T}$, auxiliary heating with electron cyclotron resonance heating (ECRH) of $2.6 \mathrm{MW}$ at $140 \mathrm{GHz}$, neutral beam injection (NBI) of $2.1 \mathrm{MW}$ with equal share of $\mathrm{H}$ and He beams, and ICRH of $4.0 \mathrm{MW}$ at $36.5 \mathrm{MHz}$. This corresponds to phase $\mathrm{E}$ in figure 1c. Such strongly ICRH-heated plasmas are known to lead to noticeable erosion of the limiter wall tiles at the outer midplane of AUG, even though recent upgrades of the ICRH antennas on AUG have been able to considerably reduce sputtering of these components as well as the core $\mathrm{W}$ concentrations [21]. The average core density was $n_{\mathrm{e}}^{\text {core }}=9-10 \times 10^{19} \mathrm{~m}^{-3}$ and electron temperature $T_{\mathrm{e}}^{\text {core }}=3.0 \mathrm{keV}$ during the 7 -s long flat-top phase of an individual He discharge. Altogether 25 identical shots were carried out such that the OSP position was varied between three poloidal locations (marked in figure 2) [22]. The overall exposure times for the three phases were $\sim 100 \mathrm{~s}, \sim 60 \mathrm{~s}$, and $\sim 10 \mathrm{~s}$, respectively. The $\mathrm{D}$ content of the plasma remained at a constant level of $\sim 10 \%$ during the experiment. The $\mathrm{H}$ and He contents showed large fluctuations due to $\mathrm{H}$ beams used for plasma heating and, on average, the He content did not increase 
much from the starting value of $80 \%$ after ICWC (see section 2). One should notice that only one week before the experiment, the $\mathrm{AUG}$ vessel was boronized with $\mathrm{B}_{2} \mathrm{D}_{6}$, resulting in significant amounts of boron in the main chamber scrape-off layer (SOL) and large influxes in the divertor region [25].

In the first part of the experiment (100 s), the OSP was poloidally set on the lowermost samples such that the pre-modified sample T2 could be exposed. This way one could investigate whether the nanoscale features would grow during the discharges. In the second phase, the OSP was moved upwards such that the sample T3 with a coral-like surface was subjected to the highest particle and power fluxes. Now the idea was to investigate erosion of existing $W$ nanostructures by ELMs as well as further modifications of the surfaces. In phase I, only type III ELMs in the $\mathrm{kHz}$ regime were produced while in phase II, type I ELMs at 120-Hz frequency were observed, resulting from reduced fuelling between the two phases. In the third phase, the OSP was further raised and small $\mathrm{N}_{2}$ blips were applied to study the impact of $\mathrm{N}$ on the nanostructures; these results will not be discussed here.

Based on Langmuir probe measurements in the vicinity of the OSP, the ion saturation flux was some $2-2.5 \times 10^{23} \mathrm{~m}^{-2} \mathrm{~s}^{-1}$ and $T_{\mathrm{e}}^{\mathrm{OSP}}=20-25 \mathrm{eV}$. Assuming that the impinging ions are mostly singly-charged $\operatorname{He}^{+}(Z=1)$, we obtain for the impact energy $E_{\text {in }}=100-150 \mathrm{eV}$ by making use of the formula $E_{\mathrm{in}}=3 Z T_{\mathrm{e}}+2 T_{\mathrm{i}}$ with the electron and ion temperatures ( $T_{\mathrm{e}}$ and $T_{\mathrm{i}}$, respectively) being approximately equal [26]. Also $\mathrm{He}^{2+}$ ions can be formed under such conditions and they would produce even higher-energy particles impinging on the surface. In any case, the impact energy is well above the threshold of $20 \mathrm{eV}$ for fuzz formation (see section 1). From the $80 \%$ He content of the plasma, the fluence during phases I and II of the experiment becomes $1-2 \times 10^{25} \mathrm{He}^{+} \mathrm{m}^{-2}$, an order of magnitude more than the $10^{24} \mathrm{He}^{+} \mathrm{m}^{-2}$ limit mentioned in section 1. The surface temperature of the samples could not be directly measured but infrared measurements from neighbouring standard wall tiles indicate $T_{\mathrm{s}}>800 \mathrm{~K}$. The samples, however, were much hotter due to their poor thermal contact with the target tiles, thus values higher than $1000 \mathrm{~K}$ are expected. As a result, all the criteria for nanostructure growth and formation were fulfilled in phases I and II during an entire discharge. On the other hand, in phase II the impact energy of helium ions during the ELMs is estimated to $E_{\mathrm{in}, \max }^{\mathrm{ELM}}=1.2-1.3 \mathrm{keV}$ by making use of the approximate formula $E_{\mathrm{in}, \max }^{\mathrm{ELM}} \approx 4.23 T_{\mathrm{e}}^{\mathrm{ped}}[26]$ with $T_{\mathrm{e}, \max }^{\mathrm{ped}} \sim 300-350 \mathrm{eV}$, and this energy is sufficient to result in significant sputtering of the nanostructures. Thus, in phase II, a competition between nanostructure growth and destruction exists.

\subsection{Results and conclusions}

The main observations can be summarized as follows:

- No noticeable net erosion has taken place, not even close to the actual OSPs, but the amount of $\mathrm{W}$ on the marker samples has increased by dozens of nanometers as a result of the plasma exposure (figure 3a). Thus, the entire OSP region is dominated 

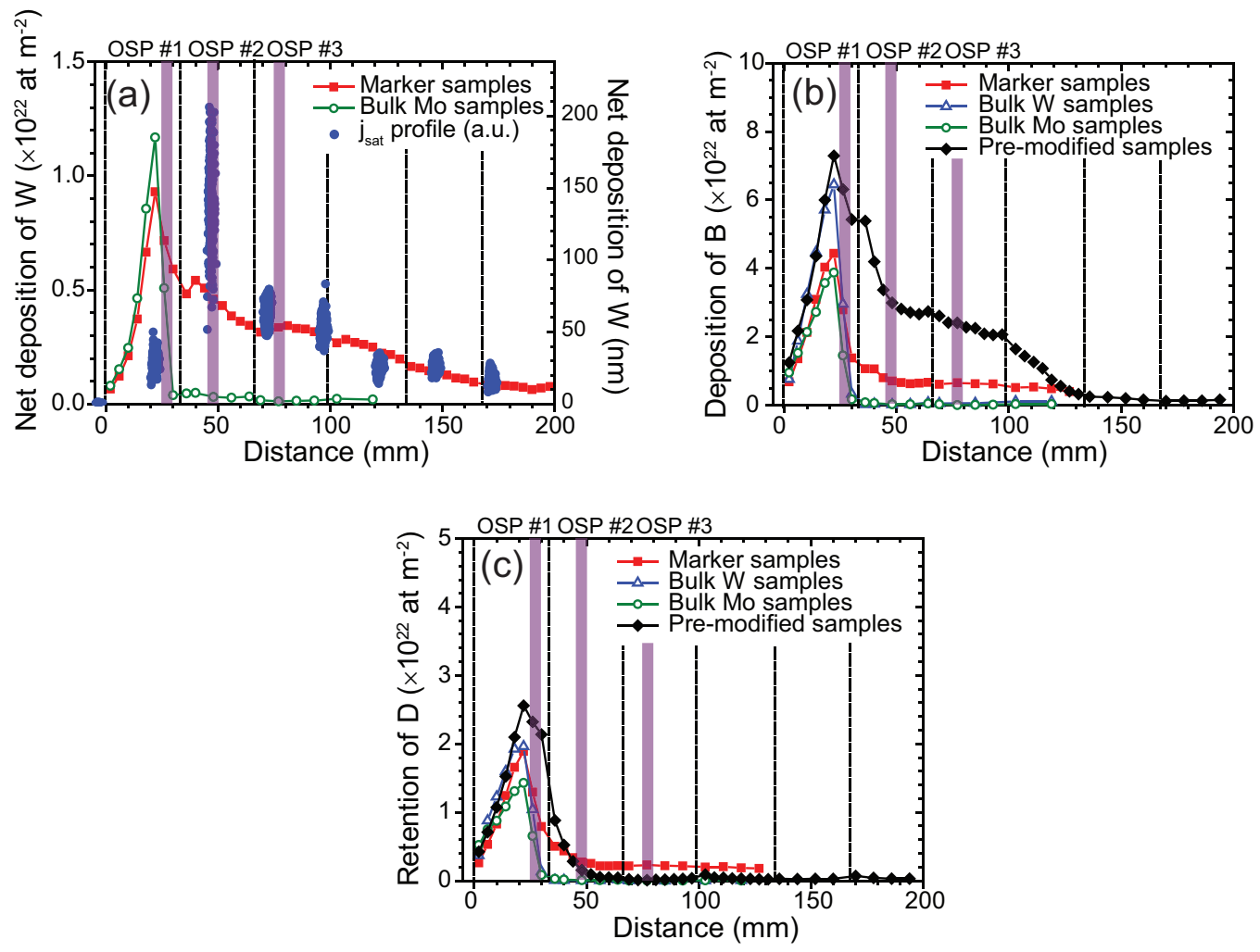

Figure 3. (a) Poloidal net deposition profile for the $\mathrm{W}$ marker sample and redeposition profile for $\mathrm{W}$ on the bulk Mo samples. For comparison, the profile for the ion saturation flux during phase II of the experiment is shown. (b,c) Poloidal deposition profiles for (b) B and (c) D on the different samples. The purple bars denote the OSP positions during the three phases of the experiment. Notice the different scales of the $y$ axes.

by net deposition with the thickest layers in the private flux region (PFR), below all the OSPs of the experiment. This is in sharp contradiction with what has been reported earlier for D plasmas: a distinct net erosion peak at the strike point and net deposition regions surrounding it [27]. Figure 3, in contrast, suggests that in helium plasmas a strong influx of material is established from the main chamber to the divertor along the magnetic field lines. There, the particles are further transported by the strong $\mathbf{E} \times \mathbf{B}$ drift towards the PFR [28]. One could argue that by poloidally shifting the OSP, the net-erosion peak formed during one phase of the experiment would have been completely removed by re-deposition during the other phases but this kind of process would not have led to the net deposition profile of figure 3 a being so smooth, without any noticeable dips or peaks around OSP\#2 or OSP\#3.

- All the samples are covered with thick co-deposited layers (up to $1 \mu \mathrm{m}$ ) consisting mainly of D, B, C, N, O, and W. The deposition profiles are qualitatively similar to the net deposition profiles for $\mathrm{W}$ in figure $3 \mathrm{a}$, as shown in figures $3 \mathrm{~b}$ and $\mathrm{c}$ for $\mathrm{B}$ and $\mathrm{D}$, respectively, but some clear differences can be observed as well. The B 
and $\mathrm{W}$ curves in figures $3 \mathrm{a}$ and $\mathrm{b}$ agree with the shape of the $\mathrm{He}^{+}$flux profile as indicated in figure $3 \mathrm{a}$ for phase II discharges while the D profile shows a peak only in the PFR for all the samples. This can be interpreted such that B and W travel to the divertor region all the way from the main chamber while $\mathrm{D}$ is embedded in the deposits from more local sources in the divertor. New experiments and careful spectroscopic measurements in the main chamber and in the divertor region are needed to verify this hypothesis.

- The rougher the sample surface, the more material is deposited on it, particularly above the OSPs. The strongest deposition is measured on the pre-modified samples with a large effective surface area while on the smooth bulk W and Mo samples deposition outside the PFR is almost non-existent. This is in line with the conclusions made in $[10,29]$ on rough surfaces favouring accumulation of material in shadowed valleys behind protruding surface peaks. The smooth Mo and bulk W surfaces respond quite differently to the impinging particles: they will be efficiently re-eroded or reflected and no noticeable deposits can form.

- For tungsten, however, the deposition peak in the PFR is almost independent of the surface roughness but relatively rough marker samples and smooth bulk Mo samples indicate net deposition being comparable (figure 3a). This can be explained by noticing that below the OSP the cold plasma conditions favour layer-by-layer growth of material without re-erosion such that the memory of the original surface is lost after a couple of seconds. On the other hand, on the pre-modified and bulk W samples much more impurities in the PFR than on the two other samples have been measured but this could be simply due to their different toroidal positions, as one can notice in figure 2.

- No signs of nanostructure growth or erosion are observed on the pre-modified samples, nor on the bulk W samples. This is attributed to the deposited layer being quickly formed on the surface and covering all the nanoscale features, thus protecting them from further exposure as SEM images of FIB-prepared cross sections on the studied samples in figure 4 illustrate. Around the OSP, the top part of a few individual corals appears to have been cut (see the tendrils at the center of the purple and yellow zoom-ins in figure 4a), possibly because they have formed leading edges and ELMs have then eroded them, while the space between the corals has been efficiently filled with deposited material. Deeper in the PFR the co-deposit can be seen to consist of $>20$ sublayers (figure $4 \mathrm{~b}$, see alternating bright and dark features inside the pit at the upper central part of the figure), coinciding with the number of discharges executed during the experiment.

We conclude that erosion of $\mathrm{W}$ surfaces and nanostructure formation or growth is overcompensated by local deposition of material in between ELMs, possibly resulting from erosion in the main chamber. As mentioned above, the situation is quite unlike the case during D operations when large net erosion is typically measured around the strike point. Different main-chamber sources are indeed plausible explanations to the 

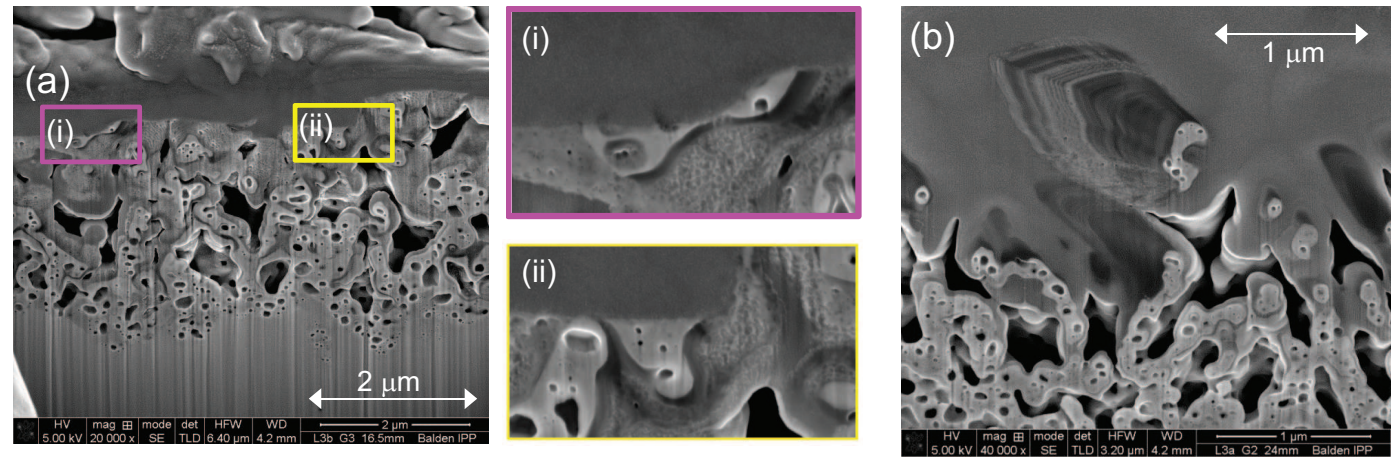

Figure 4. (a) SEM image of a FIB-prepared cross section on the sample T3 close to the OSP. The two zoom-ins (i) and (ii) of the interface between the original nanostructure and the deposited layer have been marked with purple and yellow rectangles. (b) SEM image of a FIB-prepared cross section on the sample T2 in the PFR with the layered structure of the co-deposit. Note that in all the cross sections the surface is tilted by $38^{\circ}$ with respect to the electron beam.

apparent discrepancies: in this experiment, ELMs resulted in efficient sputtering of the main-chamber wall structures, in addition to which ICRH generated energetic $\mathrm{He}^{+}$ or $\mathrm{He}^{2+}$ ions onto the limiter structures surrounding the ICRH antennas [21] in the outer midplane of AUG; in the D experiments reported in [27], only ECRH was used and no type-I ELMs were observed. However, one cannot exclude the possibility that the absence of carbon- and boron-rich surface layers around the strike-point region in deuterium plasmas is simply due to these layers being efficiently removed by chemical erosion, a process that is almost non-existent in helium.

The qualitatively different deposition patterns on the four sample types are also affected by the balance between erosion and deposition phenomena. On the smooth bulk $\mathrm{W}$ samples, the two processes are equally important while in the case of rougher samples, re-erosion is further reduced and net deposition takes over.

One additional factor contributing to the negligible growth of nanostructures during the experiment could be related to the pulsed nature of the sample exposure. As discussed in [30], the high-temperature exposure phase followed by several minutes of cooling down in between plasma discharges considerably shrinks the size of the fuzz structure on the samples. Thus, under the present exposure conditions, the fluence should have considerably higher to result in any measurable nanostructure network on tungsten.

\section{Retention of helium in tungsten samples}

The He content of selected samples was measured using foil-ERDA with $15-\mathrm{MeV}{ }^{16} \mathrm{O}^{5+}$ ions and time-of-flight ERDA with $23-\mathrm{MeV}^{127} \mathrm{I}^{6+}$ ions. In both cases, only the topmost surface layer (some 50-100 nm) could be analysed, but based on earlier studies, under low-energy and low-flux irradiations, as is the case in the AUG experiment, He is 

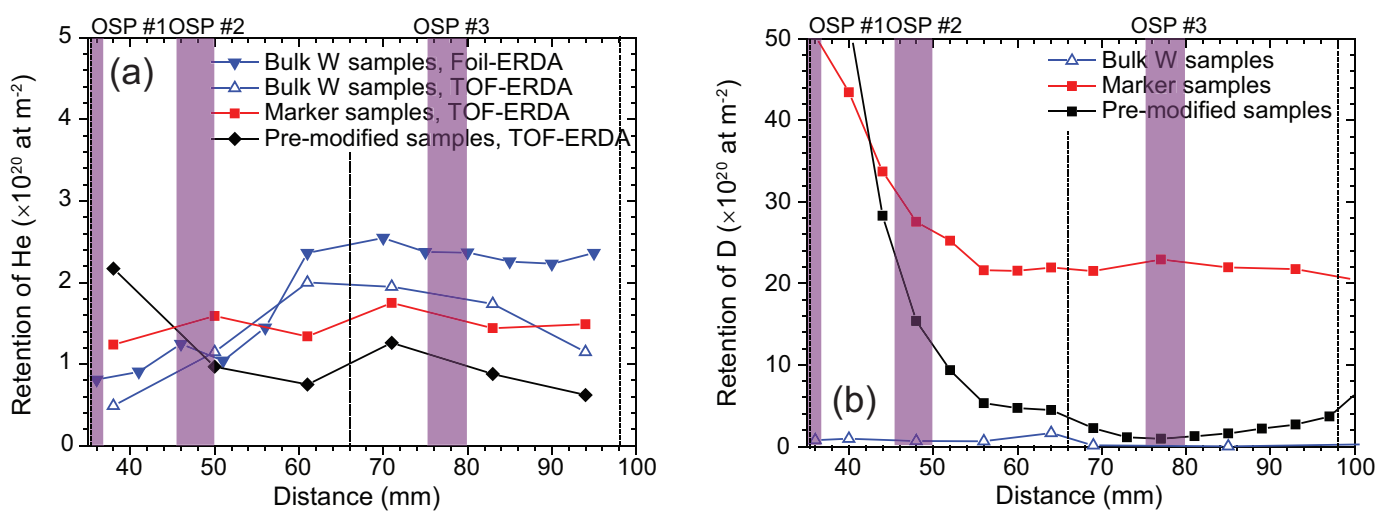

Figure 5. (a,b) Poloidal retention profiles for (a) He and (b) D on the different samples around the OSP region. Part (b) is a zoom-in of the data in figure 3c. The purple bars denote the OSP positions during the three phases of the experiment. Notice the different scales of the $y$ axes.

typically retained close to the surface due to its high probability of being trapped in defects [31]. As discussed in section 1, this will lead to the formation of a diffusion barrier in this implantation zone, which tends to limit subsequent retention of $\mathrm{H}$ and $\mathrm{D}$ in the material [7]. However, the actual accumulation of helium in W depends strongly on the exposure conditions and the surface morphology: the higher the surface temperature and the applied helium flux as well as the more porous the sample becomes due to fuzz growth, the more He is retained in the material (up to 7 at.\%) with the depth profile extending deeper than in $\mathrm{W}$ samples with a smooth surface [32]. All this influences the subsequent D and $\mathrm{H}$ retention as well.

The different ERDA profiles are shown in figure 5a for the analysed samples, located in the vicinity of the OSP, on the SOL side of it. On the bulk W samples, the He content is $0.5-2.5 \times 10^{20} \mathrm{He} \mathrm{m}^{-2}$, which is of the same order of magnitude as the surface densities measured on the $\mathrm{W}$ samples after the ICWC experiment (see section 2). The profile is also relatively flat with some point-to-point oscillations but no major increase in retention towards the PFR, as was, e.g., the case for D (see figure 5b where the data of figure $3 \mathrm{c}$ is reproduced at a higher magnification). This indicates that He indeed stays in the vicinity of the surface, either trapped in defects and pores or the topmost layer of the growing co-deposit, as proposed in [31,33]. Our results also support the idea of saturation such that the surface densities would remain $<10^{21} \mathrm{He} \mathrm{m}^{-2}$ [34].

For the pre-modified and coated marker samples, ERDA indicates the He content to be comparable to that of bulk W samples or slightly lower. However, Thermal Desorption Spectroscopy (TDS) data up to $1300 \mathrm{~K}$ suggest the total He inventory to be $10-20$ times higher on these samples (figure 6) than on bulk W, meaning that whenever the surface turns rough or porous, the penetration depth increases drastically. Interestingly, the results indicate that equally much He is accumulated on both premodified and coated samples but all the results have to be treated with caution since, on one hand, the unexposed nanostructured surface contained He from the GLADIS 

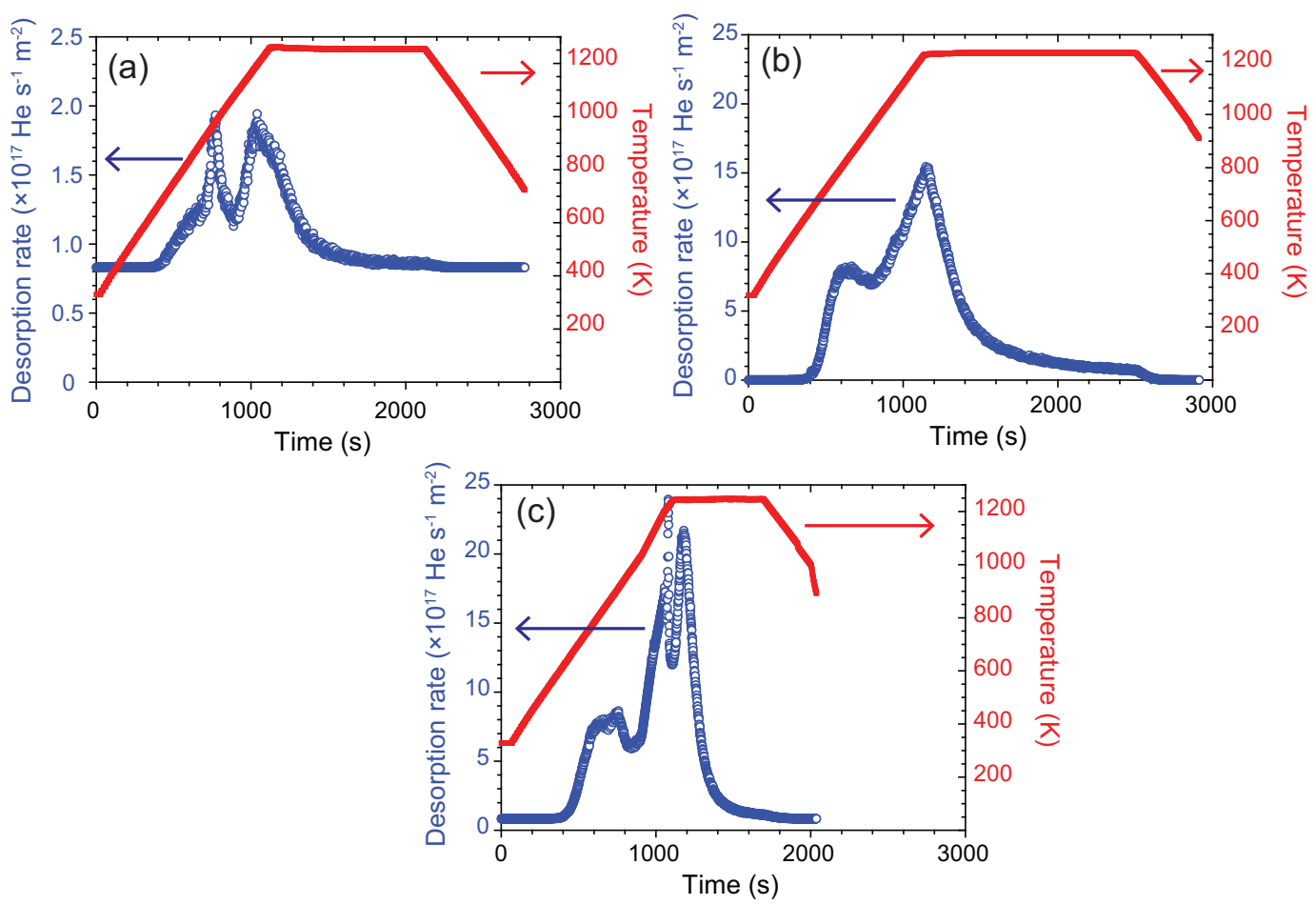

Figure 6. TDS data for the release of helium from (a) a bulk W sample, (b) a marker sample, and (c) the pre-modified sample T4, all extracted close to OSP\#3. Notice the different scales of the $y$ axes in (a), (b), and (c). The total release of helium in all the cases is: (a) $5.5 \times 10^{19}$, (b) $100 \times 10^{19}$, and (c) $81 \times 10^{19} \mathrm{He}$ atoms, respectively.

exposure and, on the other hand, a significant fraction of helium on the samples may have desorbed during the time between their exposure and analyses (up to 10 months, including cutting samples into smaller pieces). In addition, the desorption at $1300 \mathrm{~K}$ may not have been complete, meaning that not all helium retained in traps with a high binding energy is not released. Indeed, from the TDS curves one notices the qualitatively different release behaviour of helium between the different samples. On bulk W, the two peaks at $1000 \mathrm{~K}$ and $1200 \mathrm{~K}$ are comparable while from the pre-modified sample the majority of helium is released only at $1300 \mathrm{~K}$. The marker sample behaves similarly but the release at higher temperatures takes even longer, meaning either desorption from tightly-bound traps or deep from the underlying graphite substrate.

\section{Conclusions}

In this article we have studied plasma-wall interaction effects in the full-W ASDEX Upgrade during its dedicated helium campaign in 2015. Relatively clean plasmas with a He content of $>80 \%$ could be obtained by applying ICWC discharges upon changeover from $\mathrm{D}$ to He. The $\mathrm{D}$ content of the plasma then slowly decreased during the entire He campaign while the He concentration did not change much due to frequent heating of the plasmas with hydrogen NBIs. Surface analyses of W samples, however, indicated co- 
deposited layers being formed on them which contained, besides He, significant amounts of D. This can be explained by multiple erosion-deposition steps albeit globally D was released from the PFCs. One should note that the ICWC-produced deposits are some two orders of magnitude thinner than those observed after actual plasma operations in He, suggesting that ICWC is indeed effective in removing fuel and impurities from the treated wall structures.

When exposing different W samples to ELMy H-mode helium plasmas in the outer strike-point region of the divertor, no net erosion was observed but the sample surfaces had been covered with co-deposited layers. The layers were the thickest in the private flux region and extended throughout the OSP region in the case of rough and modified surfaces. Also, no clear signs of nanostructure growth or destruction could be seen. Since under D plasma operations the OSP co-incides with a region of prominent net erosion, we conclude that in He plasmas extra W sources, presumably in the main chamber, result in efficient coverage of the $\mathrm{W}$ surfaces during the first couple of seconds. However, one cannot exclude the possibility that the absence of carbon- and boron-rich surface layers in deuterium plasmas around the strike-point region is simply due to them being efficiently removed by chemical erosion.

In any case, the occurrence and growth of co-deposited layers may impact the operation of future fusion reactors. Dedicated lab experiments and modelling efforts in the presence of impurity mixes are needed to elucidate the issue further and determine its significance for the operation of ITER. Indeed, if the wall source in He plasmas is much higher than in comparable D discharges, it will be challenging to extrapolate the results from one to the other operational phase. The deposition pattern for boron observed on AUG can also be considered a proxy for beryllium in the ITER divertor since after boronization boron is predominantly deposited on the main-chamber walls. In helium plasmas, the erosion of beryllium is expected to be at least twice as strong as during D or D-T operations, meaning that thick co-deposits would be expected. Furthermore, in future fusion reactors the situation may further be altered by the operation being steady state or the individual pulses being long: the lack of high-temperature annealing phases during the period that the $\mathrm{W}$ components reside in the reactor vessel has been reported to promote the growth of thick W fuzz layers.

\section{Acknowledgements}

This work has been carried out within the framework of the EUROfusion Consortium and has received funding from the Euratom research and training programme 20142018 under grant agreement No 633053. The views and opinions expressed herein do not necessarily reflect those of the European Commission or the ITER Organization.

\section{References}

[1] Philipps V 2011 Journal of Nuclear Materials 415 S2 
[2] Brezinsek S et al 2015 Journal of Nuclear Materials 46311

[3] Neu R et al 2013 Journal of Nuclear Materials 438 S34

[4] Douai D et al 2015 Journal of Nuclear Materials 463150

[5] Baldwin M et al 2008 Nuclear Fusion 48035001

[6] Petty T J et al 2015 Nuclear Fusion $5 \mathbf{5} 093033$

[7] Ueda Y et al 2009 Journal of Nuclear Materials 386-388 725

[8] Alimov V Kh et al 2009 Physica Scripta T138 014048

[9] Baldwin M et al 2011 Nuclear Fusion 51103021

[10] Hakola A et al 2014 Physica Scripta T159 014027

[11] Kajita S et al 2009 Nuclear Fusion 49095005

[12] Kajita S et al 2011 Journal of Nuclear Materials 418152

[13] Ueda Y et al 2011 Journal of Nuclear Materials 415 S92

[14] Wright G M et al 2012 Nuclear Fusion 52042003

[15] Doerner R P et al 2016 Physica Scripta T167 014054

[16] Doerner R P et al 2011 Nuclear Fusion 51043001

[17] Douai D et al 2011 Journal of Nuclear Materials 415 S1021

[18] Douai D et al 2016 Changeover from Deuterium to Helium with Ion Cyclotron Wall Conditioning and diverted plasmas in ASDEX Upgrade Proc. 22 $2^{\text {nd }}$ Int. Conf. on Plasma Surface Interactions in Controlled Fusion Devices (Rome, Italy) p P3.116

[19] Petersson P et al 2016 Investigation of probe surfaces after ion cyclotron wall conditioning in ASDEX Upgrade Proc. 22 $2^{\text {nd }}$ Int. Conf. on Plasma Surface Interactions in Controlled Fusion Devices (Rome, Italy) p P3.118

[20] Strachan J D et al 2008 Nucl. Fusion 48105002

[21] Bobkov V et al 2017 Plasma Physics and Controlled Fusion 59014022

[22] Brezinsek S et al 2016 Erosion of He pre-exposed tungsten samples by He plasmas in the divertor manipulator of ASDEX Upgrade Proc. 22 ${ }^{\text {nd }}$ Int. Conf. on Plasma Surface Interactions in Controlled Fusion Devices (Rome, Italy) p P1.68; Nuclear Materials and Energy (in press) http://dx.doi.org/10.1016/j.nme.2016.11.002

[23] Herrmann A et al 2015 Fusion Engineering and Design 98-99 1496

[24] Greuner H et al 2011 Journal of Nuclear Materials 417495

[25] Rohde V et al 2007 Journal of Nuclear Materials 363-365 1369

[26] Guillemaut C et al 2015 Plasma Physics and Controlled Fusion 57085006

[27] Hakola A et al 2016 Physica Scripta T167 014026

[28] Hakola A et al 2016 ERO and PIC simulations of gross and net erosion of tungsten in the outer strike-point region of ASDEX Upgrade Proc. 22 ${ }^{\text {nd }}$ Int. Conf. on Plasma Surface Interactions in Controlled Fusion Devices (Rome, Italy) p P1.59; Nuclear Materials and Energy (in press) http://dx.doi.org/10.1016/j.nme.2016.09.012

[29] Schmid K et al 2015 Journal of Nuclear Materials 46366

[30] Tokitani M et al 2016 Initial growth phase of W-fuzz formation in ultra-long pulse helium discharge in LHD Proc. 22 ${ }^{\text {nd }}$ Int. Conf. on Plasma Surface Interactions in Controlled Fusion Devices (Rome, Italy) p P1.83; Nuclear Materials and Energy (in press) http://dx.doi.org/10.1016/j.nme.2016.11.023

[31] Tokunaga K et al 2003 Journal of Nuclear Materials 313-316 92

[32] Woller K B et al 2015 Journal of Nuclear Materials 463289

[33] $\mathrm{Hu} \mathrm{C}$ et al 2016 Fusion Engineering and Design 112117

[34] Hino T et al 1999 Journal of Nuclear Materials 266-269 538 